\title{
AN ANALYSIS OF SEVERAL CONCEPTS OF VIRTUALISATION
}

\author{
Anuska Sharma \\ SOEIT, Sanskriti University, Mathura, Uttar Pradesh, India
}

\begin{tabular}{|c|c|c|c|}
\hline Journal & \multicolumn{3}{|c|}{$\begin{array}{l}\text { Samvakti Journal of Research in Information Technology } \\
\text { https://www.sjrit.samvaktijournals.com } \\
\text { Volume } 2 \text { Year of Volume } 2021 \text { Page No : } 133 \text { - } 140\end{array}$} \\
\hline Discipline & \multicolumn{3}{|l|}{ Networking } \\
\hline Conference & \multicolumn{3}{|c|}{$\begin{array}{l}\text { A virtual international conference on redefining and transforming the role of higher } \\
\text { education in sustainable development }\end{array}$} \\
\hline Conference & \multicolumn{3}{|c|}{ Start Date: September 30, 2021} \\
\hline Dates & \multicolumn{3}{|c|}{ End Date : September 30, 2021} \\
\hline Institute Name & \multicolumn{3}{|c|}{$\begin{array}{l}\text { JAIN (Deemed-to-be University) in association with Council for Industrial } \\
\text { Innovation and Research }\end{array}$} \\
\hline $\begin{array}{l}\text { Date Received } \\
\text { ID } \\
\text { Dol }\end{array}$ & $\begin{array}{l}\text { : November } 21,2021 \\
: 2021.02 .26 \\
: 10.46402 / 2021.02 .26\end{array}$ & $\begin{array}{l}\text { Publication Date } \\
\text { Paper Type } \\
\text { Dol URL }\end{array}$ & $\begin{array}{l}\text { : December 11, } 2021 \\
\text { : Conference Paper } \\
\text { : https://dx.doi.org/10.46402/2021.02.26 }\end{array}$ \\
\hline \multicolumn{4}{|c|}{$\begin{array}{l}\text { Access Type : Open Access (Attribution-NonCommercial-NoDerivatives } 4.0 \text { International) } \\
\text { (C) } 2021 \text { Anuska Sharma with publication rights granted to Samvakti Journals }\end{array}$} \\
\hline
\end{tabular}

\section{ABSTRACT}

The abstraction of computer resources is referred to as virtualisation. The goal of a computing environment which is virtual is to enhance source efficiency with supplying users and programs with a single integrated operating platform depending upon on the accumulation of dissimilar and self-sufficient assets. Virtualisation at whole levels (network, storage and system) has lately resurfaced with means of improving security of system, dependability, and accessibility, lowering prices, and increasing versatility. Virtualisation" may be defined as environment with virtual machine which offers "sufficient demonstration of fundamental hardware to enable host operating systems to operate devoid of alteration. This article covers the fundamentals of system virtualisation, as well as the benefits and drawbacks of virtualisation, as well as taxonomies and difficulties.

KEYWORDS: Computer, Hardware, Software, Virtual, Virtualisation.

\section{INTRODUCTION}

Virtualisation is abstraction layer related to software that sits among hardware and operating system (OS), as well as the programs that run on top of it. Virtual machine monitor (VMM) also acknowledged as hypervisor is abstraction layer that conceals external resources related to computer system as of OS. Because VMM, rather than 
the OS, controls the hardware resources, it's feasible to execute several (potentially diverse) OSs in equivalent on identical hardware. As per consequence, physical platform is divided in 1 or additional units which are logical known as virtual machines (VMs). Only in formal world does "virtuality" vary from "reality," while having a comparable substance or impact. A virtual environment can be seen similar to actual surroundings via application programs and remaining of world in computer world, despite the fact that the underlying processes are formally different ${ }^{[1]}$.

\section{Background:}

IBM Corporation initially pioneered virtualisation in the 1960s, with the goal of partitioning a huge mainframe computer in many examples which are logical that could operate in a sole external mainframe hardware in form of host. This functionality is created to make it easier to manage the bigger mainframe systems. The scientist discovered that this partitioning feature enables many procedures and presentations to operate at similar time, improving environment's efficacy with lowering maintenance costs. Although the primary goal of this article is to give an overview of virtual security risks. Few of safety advantages which come along virtualisation are worth noting. Any virtualisation technique has two main advantages ${ }^{[2]}$.

- Sharing of resource: Dissimilar to environment which is non-virtualised, where whole assets is devoted to executing programs, in environment which is virtualised, VMs share external materials of the underlying host, including memory, storage, and network devices.

- Isolation: Isolation among machines which are virtual operating on similar external hardware is one of the most important aspects of virtualisation. Programs in one virtual machine are not able to view programs in another virtual machine.

\section{Classification:}

Lower-level functions and underlying hardware may be abstracted and isolated via virtualisation. This allows higher-level functions to be portable, as well as the distribution and accumulation of external resources. Many virtualisation methods may divided in the following categories:

\section{a. Full Virtualisation:}

The VMM are known as virtual machine manager in this method, and it executes on uppermost level of host OS, usually in form of user-space program. As a consequence, the apps and host OS operate on topmost of hardware which is virtual supplied by VMM in VMs. However, "Full Virtualisation" may be defined as a VM environment which offers "sufficient demonstration of fundamental hardware to enable guest OS for operating deprived of any alteration." In this configuration, I/O apparatuses have assigned to visitor machines with simulating external devices in monitor of virtual machine; interactions through such virtual apparatuses are subsequently routed to actual external devices, whichever by host OS driver or through VM driver. The 
primary benefit of this method is that it is very simple to implement. A typical user may install VMware Workstation similar to other package of software on their operating system of their wish. A guest OS may downloaded and executed within VMware Workstation exactly as it could operating in hardware. Major drawback of this method is low enactment, that may up to $30 \%$ lower compared to when operating on hardware straight ${ }^{[3]}$.

\section{b. OS-Layer Virtualisation:}

This idea, acknowledged as Single Kernel Image (SKI) or virtualisation which is container-based, works by executing several examples of identical OS together. It indicate that host OS, not hardware, is being virtualised. Virtualised OS image used by all of the VMs is the same. The virtualised OS image is referred to as the virtualisation layer here. These tinny structural design simplifies system organisation by allowing managers to allocate sources like memory, CPU reassurances, and disk space together while building a VM and with dynamism during execution time. OSlayer virtualisation is more efficient than other server virtualisation technologies, and it only falls short of providing the same isolation. However, there is one significant disadvantage to this approach: as VMs utilize identical kernel in form of host OS, guest OS should be identical to host OS (and as, which is impossible to execute.

\section{c. Hardware-Layer Virtualisation:}

Virtualisation on the Hardware Layer Because of its excellent virtual machine isolation and performance, this technique is widely utilized in the server industry. The VMM runs directly on hardware in this case, regulating and synchronizing guest OS access to hardware resources. Xen employs para virtualisation to offer interface of virtual machine that represents a little altered replica of primary hardware, with not virtualisable parts of $x 86$ unique set of instructionsbeing substituted by their readily equivalents which are virtualised ${ }^{[4]}$.

\section{d. Para virtualisation:}

In contrast to full virtualisation, the operating guest OS in para virtualisation must be changed for running in virtual environment. It is subtype of server virtualisation that creates squeaky interface of software among host hardware and guest OS that has been changed. The fact that guest computers is known about these are operating in environment which is virtualised is an intriguing feature of this technology. The virtual machine monitor is one of the most important features of para virtualisation technology, as it enables para virtualisation to reach enactment comparable with nonvirtualised hardware. Interaction of device in a para-virtualised environment are identical to interaction of device in fully virtualised environment; devices which are virtual in a para-virtualised environment likewise depend upon the underlying host's physical device drivers ${ }^{[5]}$. 


\section{e. Application virtualisation:}

Program virtualisation allows a user to execute server application locally exploiting local resources deprived of having to install application fully on his or her machine. Virtualised apps are created to operate in a tiny virtual environment with just the resources required to run the program. As a result of this, every single user has a virtual application environment. Amongst program and operating system of host, this tiny secluded virtual environment serves in form of layer.

\section{f. Resource virtualisation:}

Resource virtualisation is the process of virtualizing resources which are specific to system for instance "network resources, storage volumes, and name spaces, and. It may be done in a variety of ways.

- Aggregating numerous separate components into a bigger resource pool is one of them.

- Grid computing, also known as computer clusters, is a method of combining many discrete computers to create huge supercomputers with tremendous processing power.

- Dividing sole resource, like disk space, in a no. of lesser, more readily available resources of the identical type[6].

\section{g. Storage virtualisation:}

This is kind of Resource virtualisation in which logical storage is formed via extracting whole of network's actual storage resources. The resources of physical storage is first pooled for creating storage pool that is used to create logical storage. To the user, it seems in form of single monolithic device for storage since it represents aggregation of disparate physical resources.

\section{Advantages of Virtualisation:}

I. Flexibility is provided in a variety of ways. It is included because it is possible to run multiple cases of OS in single system, it is conceivable to relocate virtualised case to other external system, and virtual instances could be gracefully disconnected as of host OS using attributes such as boot 'pause, shutdown, and resume.' Virtual computers may also have their characteristics changed while they are operating, such as the amount of RAM, hard disk capacity, and so on.

II. Availability is increased since virtualised instances may continue to operate even if the real node is shut down on behalf of hardware upgrades or management. It is accomplished via temporarily relocating instances which are virtual to other system and then returning these once repairing is complete and original machine is ready for service. Hardware may be replaced, updated, repaired, and maintained devoid of any service disruption. 
III. Scalability is included since adding and removing nodes is simple. If need on behalf of volume grows with time, it's simple for adding external node to basic group installation, which can help operate current virtual machines which execute services. As a result, group would scale along with business as it grows.

IV. If multiple OS are hosted at the same time, hardware usage will almost certainly rise. It will be because virtual machines make use of hardware resources which the host operating system has left idle.

V. Security has been increased as a result of the increased separation of services. It's probable to split services via executing one service upon every virtual machine when using several virtual machines. The other services are unaffected if one is hacked. The server would have a basic setup that could support multiple virtual computers thanks to virtualisation. Each virtual machine is made up of a basic OS and 1 service, such as a web server. If it is pretended that web server has been hacked. Web pages which are hosted would be unstable, but other operating services - including file, database and mail server will not be affected ${ }^{[7]}$.

VI. Financial: By consolidating minor servers in extra powerful servers, charge savings may be realized. Hardware price drops, as well as operational price decreases in terms of people, floor space, and software licensing, all contribute to cost savings.

VII. Workload Variability: Shifting resources and priority assignments across virtual machines may readily accommodate changes in workload intensity levels. For dynamic transfer processors from 1 virtual machine to other, autonomous computing-based resource allocation methods may be employed.

VIII. Load Balancing: Because the VMM fully encapsulates software state of whole virtual machine, it is very simple to move virtual machines to different platforms for enhancing performance via improved balancing of load.

IX. Legacy Apps: If a company chooses to move to new OS, legacy applications may be executed on previous OS as guest OS inside a virtual machine. It lowers charge of migrating.

\section{Disadvantages of Virtualisation:}

I. The main disadvantage of virtualisation has been the increased overhead, which has resulted in worse performance. Due to flexibility, performance is often harmed. The engineers operated hard to reduce overhead and get it as near to the speed of a standalone real computer as possible.

II. Hardware SPOF (single point of failure) is yet a problem. Despite the fact that the virtual machine has detached from hardware, it nevertheless relies on it to function. Failure of hardware would almost certainly result in failure of the virtual computer, requiring a reboot.

III. The virtualisation platform and the management interface are inextricably connected. This may be an issue since it complicates the convergence of many platforms into a single ecosystem. 


\section{Virtualisation Challenges:}

The following are the criteria for a virtualisable architecture, according to Popek and Goldberg. A virtual machine monitor may be built for any ordinary third-generation computer if list of delicate instruction is subdivision of set of facilitated instruction. Virtual machine monitor (VMM) should have 3 characteristics listed below.

I. Efficiency Property: Allow benign commands to be executed directly on hardware without the need of VMM.

II. Resource Control Property: VMM must have full system control. While functioning systems (that operate on VMM's top) attempt for accessing resources, they must go via VMM.

III. Similarity Property: Any of program that runs on VMM's top must behave in same way as it would if VMM didn't exist.

\section{Security Vulnerabilities in Virtualisation:}

\section{a. VM Escape:}

Machines which are Virtual may share host machine's resources while maintaining segregation amongst VMs and among host and VMs. It means, machines which are virtual is built in such manner that program executing in one is not allowed to monitor or interact with program executing in another virtual machines or programs operating on host. In practice, however, organizations negotiate with segregation. They set up adjustable segregation to suit organization's requirements, taking advantage of the systems' security. Isolation has already been compromised by new software vulnerabilities. VM escape is an example of this kind of assault. If isolation among host and VMs is broken, one of the worst cases is VM escape. Application operating in virtual machine may overcome hypervisor layer and get access to host system via VM escape. Because the host computer is the root, any application that gets access to it also receives root rights, thus bypassing the virtual machine privileges. As a consequence, the environment's security architecture has completely collapsed. The host/guest interaction may be appropriately configured to address this issue.

\section{b. Denial of Service:}

Physical resources including memory disk, CPU, and network resource are shared by guest machines and underlying host in virtual machine architecture. As a result, a visitor may launch a denial-of-service attack counter to other guests on identical server. In a virtual environment, a denial of service attack is defined as an assault in which guest machine exploits entire resources of system. As a result, the system refuses service to other visitors who have made resource requests since there is no resource accessible for them. The easiest way to keep a visitor from using all of the resources is by restricting resources available to them. Virtualisation solutions now provide a method for allocating resources to every guest computer in environment. As a result, underlying virtualisation technology must be correctly arranged, avoiding a 
denial of service attack by preventing 1 guest from exploiting entire accessible resources.

\section{DISCUSSION}

In computing, virtualisation-based solutions have become commonplace. They offer a simple foundation for scalable, high-availability services, but they also bring additional security concerns. Security issues in server platforms have traditionally been discussed in stand-alone (non-virtualised) settings. The debate about cloud and virtualised platforms centers on the shared use of resources and the absence of infrastructure management. However, the effect virtualisation technologies may have on host system attack mitigation measures is often overlooked. As a result, this study looks at the security concerns and difficulties that come with moving from standalone systems to virtualised settings.

\section{CONCLUSION}

Virtualisation technology allows you to run 2 or additional OS on single machine, thereby saving you money. Some of security vulnerabilities in virtual machine environment are discussed in this article. Some of the dangers described here may be seen as advantages in some circumstances, but they are provided here to emphasize the need of exercising caution while developing and implementing the virtual environment. The abstraction of computer resources is referred to as virtualisation. The goal of a virtual computing environment is enhance resource efficiency through supplying users and programs with a single integrated operating platform depending upon the accumulation of diverse and self-governing resources. Virtualisation at entire levels (system, storage, and network) has lately resurfaced as a means of improving security of system, dependability, and availability, lowering charges, and increasing tractability. The fundamentals of virtualisation are explained in this article. 


\section{REFERENCES:}

[1] F. Douglis and O. Krieger, "Virtualisation," IEEE Internet Computing. 2013, doi: 10.1109/MIC.2013.42.

[2] N. M. M. K. Chowdhury and R. Boutaba, "A survey of network virtualisation," Comput. Networks, 2010, doi: 10.1016/j.comnet.2009.10.017.

[3] M. Klement, "Models of integration of virtualisation in education: Virtualisation technology and possibilities of its use in education," Comput. Educ., 2017, doi: 10.1016/j.compedu.2016.11.006.

[4] I. Khan, F. Belqasmi, R. Glitho, N. Crespi, M. Morrow, and P. Polakos, "Wireless sensor network virtualisation: A survey," IEEE Communications Surveys and Tutorials. 2016, doi: 10.1109/COMST.2015.2412971.

[5] J. Van De Belt, H. Ahmadi, and L. E. Doyle, "Defining and Surveying Wireless Link Virtualisation and Wireless Network Virtualisation," IEEE Commun. Surv. Tutorials, 2017, doi: 10.1109/COMST.2017.2704899.

[6] B. Yi, X. Wang, K. Li, S. K. Das, and M. Huang, "A comprehensive survey of Network Function Virtualisation," Computer Networks. 2018, doi: 10.1016/j.comnet.2018.01.021.

[7] Z. Khalid, N. Fisal, and M. Rozaini, "A survey of middleware for sensor and network virtualisation," Sensors (Switzerland). 2014, doi: 10.3390/s141224046. 